\title{
Protocolo unificado para el tratamiento transdiagnóstico de los trastornos emocionales en adolescentes a través de internet (iUP-A): Aplicación web y protocolo de un ensayo controlado aleatorizado
}

\author{
Bonifacio Sandín ${ }^{1}$, Rosa M. Valiente ${ }^{1}$, Julia García-Escalera ${ }^{1}$, David Pineda ${ }^{1}$, Victoria Espinosa ${ }^{2}$, \\ Ana M. Magaz ${ }^{3}$ y Paloma Chorot ${ }^{1}$ \\ ${ }^{1}$ Universidad Nacional de Educación a Distancia, Madrid, España \\ ${ }^{2}$ Centro Clínico Noguerol, Madrid, España \\ ${ }^{3}$ Hospital Gregorio Marañón, Madrid, España
}

\begin{abstract}
Internet-delivered unified protocol for transdiagnostic treatment of emotional disorders in adolescents (iUP-A): Web application and study protocol for a randomized controlled trial. A characteristic of transdiagnostic treatment is that it targets simultaneously the core symptoms and underlying vulnerabilities of several disorders. The UP-A is a new empirical supported cognitive-behavioral therapy (CBT) protocol developed by Ehrenreich-May's group for the transdiagnostic treatment approach of emotional disorders (i.e., depression, anxiety and related disorders) in adolescents (Ehrenreich-May et al., 2018), to be applied in a face-to-face format. Internet-delivered psychological treatments represent an emerging model to improve access to evidencebased CBT. A first aim of this study is to describe the development of a web application named Aprende a Manejar Tus Emociones (AMTE; Learn to Manage Your Emotions), designed to implement the UP-A in order to be delivered via internet (iUP-A). A second objective is to test the efficacy of the iUP-A for the treatment of depression and anxiety disorders compared to waiting list, in a randomized controlled trial. We investigated changes in a broad range of primary, secondary and transdiagnostic outcome measures.
\end{abstract}

Keywords: Transdiagnostic; UP-A; iUP-A; internet-delivered CBT; AMTE; anxiety; depression.

Resumen: Una característica del tratamiento transdiagnóstico consiste en que aborda simultáneamente los síntomas principales y las vulnerabilidades que subyacen a varios trastornos. El UP-A es un nuevo protocolo de terapia cognitivo-conductual (TCC) basada en la evidencia desarrollado por el grupo de Ehrenreich-May para el tratamiento transdiagnóstico de los trastornos emocionales (i.e., depresión, ansiedad y trastornos relacionados) en adolescentes (Ehrenreich-May et al., 2018), para ser aplicado en un formato cara-a-cara. Los tratamientos psicológicos dispensados a través de internet representan un modelo emergente para mejorar el acceso a la TCC basada en la evidencia. Un primer objetivo del presente estudio consiste en describir el desarrollo de una aplicación web denominada Aprende a Manejar Tus Emociones (AMTE), diseñada para implementar el UP-A con objeto de ser dispensado a través de internet (iUP-A). Un segundo objetivo es probar la eficacia del iUP-A para el tratamiento de la depresión y los trastornos de ansiedad en comparación con una lista de espera, en un ensayo controlado aleatorizado. Se investigan los cambios en un amplio rango de medidas de resultado primarias, secundarias y transdiagnósticas.

Palabras clave: Transdiagnóstico; UP-A; iUP-A; TCC por internet; AMTE; ansiedad; depresión.

Recibido: 14 de julio de 2019; aceptado: 15 de agosto de 2019 Correspondencia: Paloma Chorot, Facultad de Psicología, Juan del Rosal 10, 28040 Madrid, España. Correo-e: pchorot@psi.uned.es Agradecimientos: El estudio forma parte de un proyecto I+D+i financiado por el Ministerio de Economía y Competitividad, Ref. PSI201344480-P, concedido a BS, PC y RMV. Agradecemos a Jill Ehrenreich-May, Professor of Psychology de la Miami University (Coral Gables, US), su ayuda facilitándonos materiales del UP-C y su curso formativo impartido en Madrid (2016) sobre la aplicación de los protocolos UP-A y UP-C. Agradecemos a Cristina Botella, catedrática de la Universidad Jaime I (Castellón, España), su apoyo permanente en lo concerniente al diseño y construcción de la plataforma AMTE, y a Diana Castilla (profesora de la Universidad de Valencia, Valencia, España), sus valiosas aportaciones durante el diseño de la plataforma. Agradecemos a Raquel Viejo, del Centro de Medios Audiovisuales (CEMAV) de la UNED, su participación en la producción de los vídeos incluidos en la aplicación. Estamos especialmente agradecidos a Manuel Ottaviano (Universidad Politécnica de Madrid) y a Carlos de la Sierra (Rotor Studios, Sydney, Australia) por llevar a cabo los trabajos informáticos para el desarrollo de la aplicación, habiendo realizado, respectivamente, el diseño de software del sistema y el diseño gráfico del sistema. 


\section{Introducción}

Los trastornos de ansiedad se encuentran entre los trastornos mentales más comunes durante la adolescencia. Una de las más recientes publicaciones a este respecto de la National Comorbitidy Survey (Kessler et al., 2012) indicó una tasa de prevalencia anual del 25\% para los trastornos de ansiedad en la población adolescente, tasa muy superior a la de cualquier otro tipo de trastorno mental (seguido de los trastornos exteriorizados [16.3\%] y la depresión [10\%]). Aunque se han referido tasas muy variadas en la literatura, la mayor parte de la evidencia indica que los trastornos de ansiedad son los más prevalentes durante la infancia y la adolescencia (véase Sandín, Chorot y Valiente, 2018). Aunque los estudios epidemiológicos han proporcionado datos dispares con variaciones entre el 8.3 y el $32.4 \%$ para las tasas de «cualquier trastorno de ansiedad» (Sandín et al., 2018), uno de los trastornos de ansiedad más prevalentes durante la adolescencia es la fobia social (Chavira y Stein, 2005; Sandín, 1997), sugiriendo algunos autores que la prevalencia de la ansiedad social y del trastorno de ansiedad social se duplica al pasar de la infancia a la adolescencia (Essau, Conradt y Petermann, 1999). Una cuestión importante es que, aparte de los trastornos de ansiedad, los síntomas subclínicos de ansiedad (especialmente los miedos) son fenómenos muy comunes durante la infancia y la adolescencia (Essau et al., 1999; Valiente, Sandín y Chorot, 2003).

Los trastornos de ansiedad y los trastornos depresivos se han relacionado con elevadas tasas de comorbilidad entre sí y con otros trastornos mentales. En general, los estudios epidemiológicos han indicado que las semejanzas entre los trastornos de ansiedad y la depresión son mayores que las diferencias (Sandín, Chorot y Valiente, 2012), y que la comorbilidad con otros trastornos es muy elevada, habiendo oscilado en algunos estudios entre el 40\% y el 80\% (Wittchen et al., 2011). La evidencia clínica y epidemiológica suele indicar que más del 50\% de los individuos con diagnóstico de depresión también presentan al menos un trastorno de ansiedad, y viceversa, más del $50 \%$ de los individuos con diagnóstico primario de algún trastorno de ansiedad también reúne los criterios de algún trastorno depresivo (Mineka, Watson y Clark, 1998). Así mismo, la comorbilidad entre los síntomas de los trastornos de ansiedad y los síntomas depresivos también suele ser elevada (Sandín et al., 2018; Sandín, Chorot, Valiente y Chorpita, 2010).

A pesar de ello, la mayor parte de los tratamientos psicológicos de los trastornos emocionales se dirigen a trastornos específicos, actuando generalmente a espaldas de la elevada comorbilidad que se da entre dichos trastornos. Es cierto que actualmente existen buenos protocolos manualizados de terapia cognitivo-conductual (TCC) basada en la evidencia para el tratamiento de los principales trastornos emocionales (especialmente para los trastornos de ansiedad) (Moriana y Martínez, 2011; Nathan y Gorman, 2015). La TCC ha progresado enormemente siguiendo una aproximación específica (i.e., centrada en trastornos específicos), basándose en modelos etiopatogénicos que corresponden igualmente a trastornos concretos (p.ej., actualmente existen modelos separados o teorías específicas para todos y cada uno de los trastornos de ansiedad). Estos modelos han sido de gran utilidad para el conocimiento y explicación de los trastornos mentales, así como también para el avance y perfeccionamiento de la TCC.

La terapia basada en la evidencia dirigida a trastornos específicos presenta importantes limitaciones, especialmente cuando se aplica a los trastornos emocionales. Su principal limitación consiste en que, al dirigirse a un trastorno específico, no aborda el problema de la comorbilidad, por lo que generalmente se requiere la aplicación secuencial de varios protocolos a un mismo paciente, pues lo más común es que este reúna los criterios para el diagnóstico de otros trastornos emocionales comórbidos. El hecho de tener que aplicar diversos protocolos y manuales de tratamiento a un mismo paciente dificulta significativamente la práctica clínica, especialmente en los servicios donde el tiempo disponible es más limitado (p.ej., en los servicios públicos de salud mental), siendo esto un obstáculo por el coste que suele suponer. Aunque existen buenos protocolos específicos, un problema serio viene dado por las diversas barreras asociadas a su aplicación en los centros clínicos (Kazdin y Blase, 2011), entre las cuales se incluyen los costes para el paciente (tanto económicos como por el tiempo requerido), la carencia de profesionales formados en la aplicación de los diferentes protocolos, y las dificultades de utilizarlos en la práctica clínica rutinaria. Lo cual redunda en una pobre diseminación de los protocolos de intervención basados en la evidencia, y en una pobre atención psicológica especializada.

Recientemente se ha propuesto un enfoque psicológico innovador para abordar el tratamiento de los trastornos emocionales basado en el transdiagnóstico (para una presentación comprensiva sobre el transdiagnóstico, véase Sandín et al., 2012; Sandín, 2012). Desde el punto de vista psicopatológico, el transdiagnóstico consiste en entender los trastornos mentales sobre la base de un rango de procesos cognitivos y conductuales etiopatogénicos causales y/o mantenedores comunes a grupos consistentes de trastornos mentales. A este respecto, el transdiagnóstico, aunque se basa en una concepción di- 
mensional de la psicopatología, consiste más en una integración entre ambos enfoques que en un rechazo de la clasificación categorial. Actualmente se han referido en la literatura numerosos factores que podrían actuar como procesos transdiagnósticos comunes a los trastornos emocionales, entre los que se incluyen el perfeccionismo, el afecto positivo y negativo, la atención autofocalizada, la incontrolabilidad/impredecibilidad, la evitación emocional, la intolerancia a la incertidumbre, la sensibilidad a la ansiedad, las diversas estrategias de regulación emocional, y distintos procesos relacionados con la atención, la memoria el razonamiento, el pensamiento y la conducta (Sandín, et al., 2012).

La TCC basada en el transdiagnóstico, i.e., TCCtransdiagnóstica (TCC-T), se centra en los conceptos psicopatológicos transdiagnósticos comunes a diversos trastornos, y su progreso ha implicado el desarrollo de nuevos protocolos de tratamiento no focalizados en trastornos específicos sino en conjuntos de trastornos (p.ej., los trastornos emocionales), dando lugar a protocolos integrados o unificados de intervención. Partiendo de este enfoque, la TCC-T ha sido definida como una forma de terapia destinada a individuos que presentan múltiples diagnósticos (elevada comorbilidad), no a individuos con un solo trastorno, y ha sido caracterizada por: (a) focalizarse en procesos cognitivos, conductuales y fisiológicos que son compartidos o comunes a través de los diferentes trastornos (i.e., denominados factores unificadores), y (b) adoptar un enfoque científico convergente o integrativo (Clark, 2009; Pascual-Vera y Belloch, 2018; Mansel et al., 2009; Sandín et al., 2012). Por tanto, la TCC-T se basa en las características psicopatológicas que son comunes a trastornos (o síntomas) e individuos, no en las características diferenciales y propias de un trastorno o un individuo concreto (tratamiento individualizado). Obviamente esto no implica que la TCC-T no pueda aplicarse individualmente (Rosique, Sandín et al., 2019), pues lo esencial para que sea un tratamiento transdiagnóstico no es su forma de aplicación individual o grupal, sino que la intervención aborde primariamente procesos etiopatogénicos (factores causales de vulnerabilidad y/o mantenimiento) comunes a más de un trastorno (p.ej., común a los trastornos emocionales). Por ello debe conocerse la implicación etiológica de tales procesos o mecanismos transdiagnósticos en el conjunto de los trastornos correspondientes ${ }^{1}$.

\footnotetext{
${ }^{1}$ Aunque este es un aspecto central de la TCC-T, tal vez por lo atractivo de este nuevo enfoque, desafortunadamente se está produciendo un abuso y uso incorrecto del concepto de transdiagnóstico, y especialmente en lo que concierne a la consideración de un tratamiento como tratamiento transdiagnóstico propiamente dicho. Esto perturba
}

Algunos estudios de meta-análisis que han evaluado de forma rigurosa la eficacia de la TCC-T (Andersen, Toner y Bland, 2016; García-Escalera et al., 2016) sugieren que el transdiagnóstico posee un efecto equivalente a la TCC focalizada en problemas específicos, si bien con las ventajas asociadas al enfoque transdiagnóstico. Aunque existen diversos protocolos para el tratamiento transdiagnóstico de los trastornos emocionales, el que posee mayor grado de aceptación, consistencia teórica y validación empírica es el protocolo unificado (UP) elaborado por Barlow et al. (2011) para población adulta (p.ej., Grill, Castañeiras y Fasciglione, 2017; Rosique, Chorot et al., 2019; para una revisión de meta-análisis, véase Sakiris y Berle, 2019).

La mayor parte de los protocolos de TCC-T han sido diseñados para ser aplicados en población adulta. Partiendo del protocolo unificado (UP) de Barlow et al. (2011), Ehrenreich-May et al. (2018; Ehrenreich-May, Bilek, Queen y Hernández-Rodríguez, 2012) desarrollaron dos versiones del UP para ser aplicados a la población infantojuvenil: (1) el Unified protocol for transdiagnostic treatment of emotional disorders in children (UP-C), y (2) el Unified protocol for transdiagnostic treatment of emotional disorders in adolescents (UP-A). Los protocolos pueden ser aplicados a niños (UP-C) o adolescentes (UP-A) con múltiples tipos de trastornos emocionales, ya que no se focalizan en ningún tipo específico de trastorno emocional. Ambos protocolos consisten en un tratamiento cognitivo-conductual transdiagnóstico focalizado en la emoción y en la regulación emocional que integra técnicas de intervención cognitivo-conductual basadas en la evidencia, estando especialmente indicado para niños y adolescentes que presentan múltiples trastornos emocionales o síntomas mixtos o subclínicos de varios trastornos emocionales. La inter-

\footnotetext{
y enturbia el desarrollo científico de este nuevo enfoque, al considerar como tal cualquier tipo de tratamiento que pueda tener efectos genéricos, tales como el mindfulness, la psicoterapia psicodinámica, la terapia de aceptación y compromiso, etc. (Sauer-Zavala et al., 2017). El hecho de que un tratamiento tenga un efecto genérico sobre múltiples trastornos no significa que sea un tratamiento transdiagnóstico. Este error puede observarse, por ejemplo, en el reciente estudio de meta-análisis de Newby et al. (2015) sobre la eficacia del tratamiento psicológico transdiagnóstico en los trastornos emocionales; de los 47 estudios examinados en este meta-análisis, sólo 15 fueron incluidos en el posterior meta-análisis llevado a cabo por García-Escalera, Chorot, Valiente, Reales y Sandín (2016) sobre el mismo tema. El meta-análisis de Newby et al. (2015) es una especie de «totum revolutum», pues mezcla estudios con enfoques heterogéneos que no pueden ser considerados como tratamientos transdiagnósticos, basados, p.ej., en protocolos clásicos de TCC aplicados a grupos de trastornos, protocolos híbridos, protocolos individualizados, o terapias psicológicas meramente genéricas (mindfulness, psicoterapia psicodinámica, etc.).
} 
vención aborda mecanismos comunes a los trastornos emocionales (trastornos de ansiedad, trastornos depresivos, y otros trastornos relacionados) que están implicados en la elevada comorbilidad, especialmente los relacionados con el neuroticismo o afecto negativo (variable temperamental implicada en el desarrollo de los trastornos emocionales). Los niños y adolescentes con elevados niveles de afecto negativo tienden a experimentar las emociones negativas con elevada intensidad, tales como el miedo, la ansiedad, la tristeza o la ira, y a sentirse con excesivos niveles de malestar emocional. Por ello, uno de los principales objetivos de estos protocolos consiste en reducir estas respuestas, así como también el malestar emocional asociado, lo cual se lleva a cabo mediante estrategias cognitivo-conductuales dirigidas a (1) incrementar la flexibilidad cognitiva, (2) identificar y prevenir la evitación emocional y los comportamientos desadaptativos impulsados por emociones, (3) incrementar la conciencia de las emociones centradas en el presente y la aceptación de las sensaciones físicas relacionadas con emociones desagradables, (4) incrementar las conductas positivas, y (5) facilitar la exposición a desencadenantes interoceptivos y situacionales. Aunque estas estrategias son consistentes con las aplicadas mediante terapia cognitivo-conductual focalizada en trastornos específicos, su aplicación se centra en aspectos psicopatológicos primarios (nucleares) y genéricos relacionados con las respuestas desadaptativas a las emociones negativas en general.

Se ha constatado evidencia empírica sobre la eficacia de estos nuevos protocolos, i.e., tanto sobre la eficacia del UP-A (Ehrenreich-May et al., 2017; Queen, Barlow y Ehrenreich-May, 2014) como sobre la eficacia del UP-C (Ehrenreich-May y Bilek, 2012; Kennedy, Bilek y Ehrenreich-May, 2019. A partir de estos estudios se desprende que tales protocolos de TCC-T son al menos tan eficaces para tratar la ansiedad y la depresión como los protocolos específicos de TCC validados empíricamente, y además producen ciertas ganancias adicionales relacionadas con reducciones de la reactividad emocional y mejoras en la regulación emocional.

Nuestro grupo de investigación de la Universidad Nacional de Educación a Distancia (UNED, España) ha adaptado recientemente el UP-A aplicándolo en un contexto de prevención universal para la prevención de la ansiedad y la depresión en adolescentes (García-Escalera et al., 2017). El protocolo fue aplicado en formato grupal en el ámbito escolar (adolescentes entre 13 y 17 años de edad). Tanto los datos procedentes de un ensayo abierto (García-Escalera et al., 2019), como los obtenidos a partir de un ensayo controlado aleatorizado (García-Escalera et al., 2020), indican que el protocolo resul- tó eficaz para reducir los síntomas de ansiedad y depresión en los participantes con niveles de sintomatología subclínica (i.e., prevención indicada). También fue eficaz para reducir la interferencia asociada a la ansiedad y la depresión, y para disminuir la gravedad de los principales problemas descritos por cada adolescente.

Aunque la TCC aplicada cara-a-cara, bien en formato individual o grupal, ha demostrado ser eficaz para el tratamiento de los trastornos emocionales, no está exenta de algunos problemas importantes. Este tipo de terapia, como cualquier terapia aplicada de esta manera, puede resultar particularmente gravosa (en tiempo y coste económico), y puede generar un elevado grado de estigmatización, particularmente en población adolescente. Puede también asociarse a otras barreras, como la distancia (p.ej., personas que viven en zonas rurales) o la falta de profesionales cualificados. Una forma de terapia emergente, desarrollada especialmente durante la última década, es la TCC aplicada a través de internet (iTCC) (para una revisión, véase Andersen, 2016). La iTCC posee ciertas ventajas potenciales sobre la TCC aplicada cara-a-cara, tales como facilitar el acceso físico al tratamiento, reducir el coste económico, minimizar el estigma, reducir el tiempo de atención del terapeuta, maximizar la relación coste-efectividad, mejorar el apoyo continuado al paciente $y$, en último término, incrementar en la población el acceso a los tratamientos psicológicos basados en la evidencia. Se ha demostrado que la iTCC es eficaz y coste-efectiva para los trastornos de ansiedad y la depresión media o moderada, y de que no es inferior a los tratamientos de TCC validados empíricamente (Andersson, Titov, Dear, Rozental y Carlbring, 2019). Un problema es que la iTCC se ha aplicado generalmente focalizada en trastornos específicos siguiendo la orientación clásica de la TCC, siendo limitado el número de estudios basados en el nuevo enfoque transdiagnóstico de los trastornos emocionales. Aun así, diversos estudios han constatado que la iTCC transdiagnóstica (iTCC-T) posee niveles de eficacia sobre los trastornos emocionales (ansiedad y depresión) similares a los referidos para la iTCC (Anderson et al., 2019; García-Escalera et al., 2016).

Uno de los principales grupos investigación sobre la iTCC-T aplicada a los trastornos emocionales es el grupo de Titov (Macquarie University, Australia) (Titov, Dear, Johnston y Terides, 2012), el cual ha proporcionado amplia evidencia sobre la eficacia de esta modalidad de intervención para el tratamiento de los trastornos emocionales en población adulta. En España, el grupo de Botella (Universidad Jaime I de Castellón, España) puso en marcha el primer programa de iTCC-T (programa transversal de regulación emocional), dirigido al tratamiento de los 
trastornos emocionales en adultos (González-Robles et al., 2015). Que nosotros sepamos, aún no se ha publicado ningún protocolo de iTCC-T para el tratamiento de los trastornos emocionales en niños y adolescentes.

Uno de los objetivos de nuestro grupo de investigación de la UNED ha sido la adaptación del protocolo UP-A para ser aplicado a través de internet en población de habla española. Para ello, en primer lugar era necesario elaborar una aplicación web que nos permitiera administrar el tratamiento mediante un ordenador personal o una tableta electrónica. Con esta finalidad, durante estos últimos años hemos llevado a cabo el desarrollo del programa Aprende a Manejar Tus Emociones (AMTE), una aplicación web para adaptar el protocolo UP-A a un formato aplicable a través de internet (i.e., el iUP-A). AMTE es un programa interactivo auto-administrado vía internet diseñado para el tratamiento de los trastornos emocionales en adolescentes, para lo cual incluye la implementación del iUP-A.

Un segundo objetivo es llevar a cabo un ensayo controlado aleatorizado (ECA) con objeto de probar la eficacia del iUP-A en una muestra de adolescentes con trastornos o síntomas elevados de ansiedad y depresión. Hipotetizamos que el grupo experimental (aplicación del protocolo iUP-A) debería experimentar mayor mejoría que el grupo de control (lista de espera) en las variables de ansiedad y depresión, así como también en un amplio rango de variables transdiagnósticas. Estos cambios deberían mantenerse significativos durante el post-tratamiento y durante las fases de seguimiento (a los 3-6-y 12-meses). También se pretende examinar la viabilidad, usabilidad y aceptabilidad del programa. En el presente artículo se presenta la aplicación web y el protocolo de estudio del ECA.

\section{Aplicación web: Aprende a Manejar Tus Emociones (AMTE)}

\section{La plataforma Aprende a Manejar Tus Emociones} (AMTE) es una aplicación basada en internet que incluye la implementación del iUP-A a través de la cual se lleva a cabo la intervención; su utilización es compatible con ordenador personal y tableta electrónica. Contiene los materiales y funciones necesarios para la aplicación del iUP-A, incluyendo los vídeos didácticos, las hojas de ejercicios y tareas, el texto de los avatares a través de diversos tipos de viñetas, y las diferentes herramientas. Su estructura está basada en la plataforma web desarrollada por el grupo de Botella (González-Robles et al., 2015) para el tratamiento transdiagnóstico de los trastornos emocionales en adultos vía internet. BS, PC y RMV son responsables de la aplicación, habiendo lleva- do a cabo su diseño y la elaboración de sus contenidos (protocolos de evaluación, vídeos, ejercicios, viñetas, tareas, herramientas transversales e intramodulares, etc.). JGE ha colaborado en las revisiones y correcciones durante las pruebas finales de funcionamiento del protocolo.

La aplicación incluye tres portales, i.e., un portal principal (portal usuario) y dos portales complementarios (portal padres y portal terapeuta). El portal usuario integra los componentes principales del programa a través de cinco herramientas básicas: Módulos, Tareas, ¿Cómo estoy?, Diario, y Mis progresos. Incluye también los protocolos de evaluación pre-tratamiento, post-tratamiento y seguimiento.

\section{Protocolo de evaluación pre-tratamiento/post- tratamiento y seguimiento}

Este protocolo de evaluación se aplica justo antes de comenzar con el primer módulo (evaluación pre-tratamiento), justo después de finalizar el último módulo (evaluación post-tratamiento) y durante el seguimiento (a los 3-, 6- y 12-meses de finalizar el tratamiento). En todos los casos se incluyen los siguientes instrumentos de evaluación: RCADS, EAN, CDN, PANASN, CASI, EASI-A, SWLS-C, PSWQN, SASC-R, y PDSS-SR (véase la sección de Instrumentos de evaluación).

\section{Módulos}

La aplicación incluye los 8 módulos que se especifican en la Tabla 1, los cuales van dirigidos primariamente al tratamiento de los siguientes trastornos (o síntomatología) emocionales: trastorno de ansiedad de separación, trastorno de ansiedad social (fobia social), trastorno de ansiedad generalizada, trastorno de pánico, agorafobia, fobia específica, otros trastornos de ansiedad especificados o no especificados, trastorno depresivo mayor, trastorno depresivo persistente (distimia), y otros trastornos depresivos especificados o no especificados. Secundariamente, el programa también puede ser de ayuda complementaria para el trastorno obsesivo-compulsivo y el trastorno de estrés postraumático.

Los módulos incluyen la información nuclear del programa y mediante ellos se pretende enseñar al adolescente los conceptos básicos y necesarios para adquirir las estrategias adaptativas de regulación emocional. Cada módulo incluye varios vídeos adaptados al rango de edad de los usuarios y diversos ejercicios complementarios. Los ejercicios van dirigidos a la práctica de lo aprendido a través de los vídeos. Pueden descargarse hojas de ejercicios en formato PDF desde la propia plata- 
Tabla 1. Módulos del protocolo iUP-A incluidos en la aplicación AMTE

\begin{tabular}{|c|c|}
\hline Módulo & Contenido \\
\hline $\begin{array}{l}\text { Módulo 1: } \\
\text { Fomentando la motivación }\end{array}$ & $\begin{array}{l}\text { Identificar los problemas principales y establecer objetivos. Introducción a la estructu- } \\
\text { ra, finalidad, y funcionamiento del programa. Importancia de practicar. Descubrir qué } \\
\text { te motiva a cambiar. }\end{array}$ \\
\hline $\begin{array}{l}\text { Módulo 2: } \\
\text { Empieza a comprender tus emociones }\end{array}$ & $\begin{array}{l}\text { Psicoeducación sobre las emociones y su función. Comprender las tres partes de las } \\
\text { emociones. Entender que toda emoción tiene su antes, durante y después. Aprender } \\
\text { sobre el círculo vicioso de la evitación emocional. }\end{array}$ \\
\hline $\begin{array}{l}\text { Módulo 3: } \\
\text { Disfruta de actividades positivas }\end{array}$ & $\begin{array}{l}\text { Actividades de obligación y actividades de diversión. Fomentar las actividades positivas } \\
\text { de tiempo libre. Comprender qué es la «acción opuesta». Experimentos conductuales } \\
\text { centrados en la emoción. }\end{array}$ \\
\hline $\begin{array}{l}\text { Módulo 4: } \\
\text { Conciencia de tus experiencias emocionales }\end{array}$ & $\begin{array}{l}\text { Aprender y practicar la conciencia centrada en el presente. Escaneo del cuerpo. Tomar } \\
\text { conciencia de tus propias sensaciones. Tomar conciencia del presente sin juzgar. Prac- } \\
\text { ticar la conciencia emocional sin juzgar. }\end{array}$ \\
\hline $\begin{array}{l}\text { Módulo 5: } \\
\text { Aprende a ser flexible en tu forma de pensar }\end{array}$ & $\begin{array}{l}\text { Comprender lo que son las interpretaciones automáticas y aprender a detectarlas. } \\
\text { Aprender a conocer y detectar las «trampas de pensamiento». } \\
\text { Asociar pensamientos y conductas pensando como un detective y aplicando estrategias } \\
\text { de resolución de problemas. }\end{array}$ \\
\hline $\begin{array}{l}\text { Módulo 6: } \\
\text { Afronta tus sensaciones físicas }\end{array}$ & $\begin{array}{l}\text { Aprender a percibir las sensaciones corporales como algo natural. Comprender la aso- } \\
\text { ciación entre las emociones y las sensaciones corporales. La evitación interoceptiva. } \\
\text { Ejercicios de provocación de sensaciones corporales. }\end{array}$ \\
\hline $\begin{array}{l}\text { Módulo 7: } \\
\text { Afronta situaciones emocionales }\end{array}$ & $\begin{array}{l}\text { Comprender lo que son las situaciones emocionales y su relación con el círculo vicioso } \\
\text { de la evitación interoceptiva. Psicoeducación sobre la importancia de enfrentarse a las } \\
\text { situaciones que provocan comportamientos emocionales. Aprender a realizar y practi- } \\
\text { car planes de exposición paso-a-paso a situaciones emocionales. }\end{array}$ \\
\hline $\begin{array}{l}\text { Módulo 8: } \\
\text { Trata de mantener lo conseguido }\end{array}$ & $\begin{array}{l}\text { Revisión de las estrategias y habilidades conseguidas a lo largo del programa y valora- } \\
\text { ción de los objetivos conseguidos. Elaboración de un plan para practicar lo aprendido e } \\
\text { identificar y afrontar futuros desafíos. Prevención de recaídas. }\end{array}$ \\
\hline
\end{tabular}

forma como ayuda y control en la realización de los ejercicios.

Los módulos se aplican de modo secuencial; no es posible pasar al siguiente módulo sin haber superado el módulo actual. En cualquier momento del periodo de tratamiento, el adolescente podrá repasar los módulos anteriores. Desde el portal del terapeuta, este podrá apreciar el progreso en los diferentes módulos, incluyendo el tiempo de uso de la aplicación en cada módulo.

\section{Herramientas transversales}

El programa cuenta con las cuatro herramientas transversales siguientes: Tareas, ¿Cómo estoy? Diario, y Mis progresos. La herramienta ¿Cómo estoy? integra las dos herramientas de evaluación intra-tratamiento, i.e., la Evaluación diaria y la Evaluación semanal.

Tareas. Aparte de los ejercicios que se recomiendan al usuario en los diferentes módulos, cada módulo tiene asignadas varias tareas fundamentales para practicar lo aprendido en este y para adquirir las habilidades relacio- nadas con los contenidos del mismo. Esta es una herramienta transversal que se utiliza fuera del módulo; las tareas se alojan, dentro de esta herramienta, en 5 secciones relacionadas con cada grupo de tareas (Conócete, Disfruta, Mindfulness, Detective y Atrévete). Igual que con los ejercicios, pueden descargarse hojas en formato PDF para ayudar a la realización de las tareas. Un aspecto importante de las tareas es que estas quedan registradas en la aplicación, de tal forma que tanto el usuario como el terapeuta tendrán acceso a ellas en cualquier momento (el terapeuta podrá comprobar hasta qué punto las tareas se llevan a cabo y la calidad de estas) (véanse las Figuras 1 y 2).

Diario. La herramienta Diario se muestra en forma de calendario y proporciona información general sobre las actividades del usuario, especialmente en lo que concierne a la realización de las tareas.

Evaluación diaria. Esta herramienta posibilita llevar a cabo una evaluación sobre el estado emocional de la persona y sobre la actividad física, referidos al último día. El estado emocional se evalúa según las dimensiones 

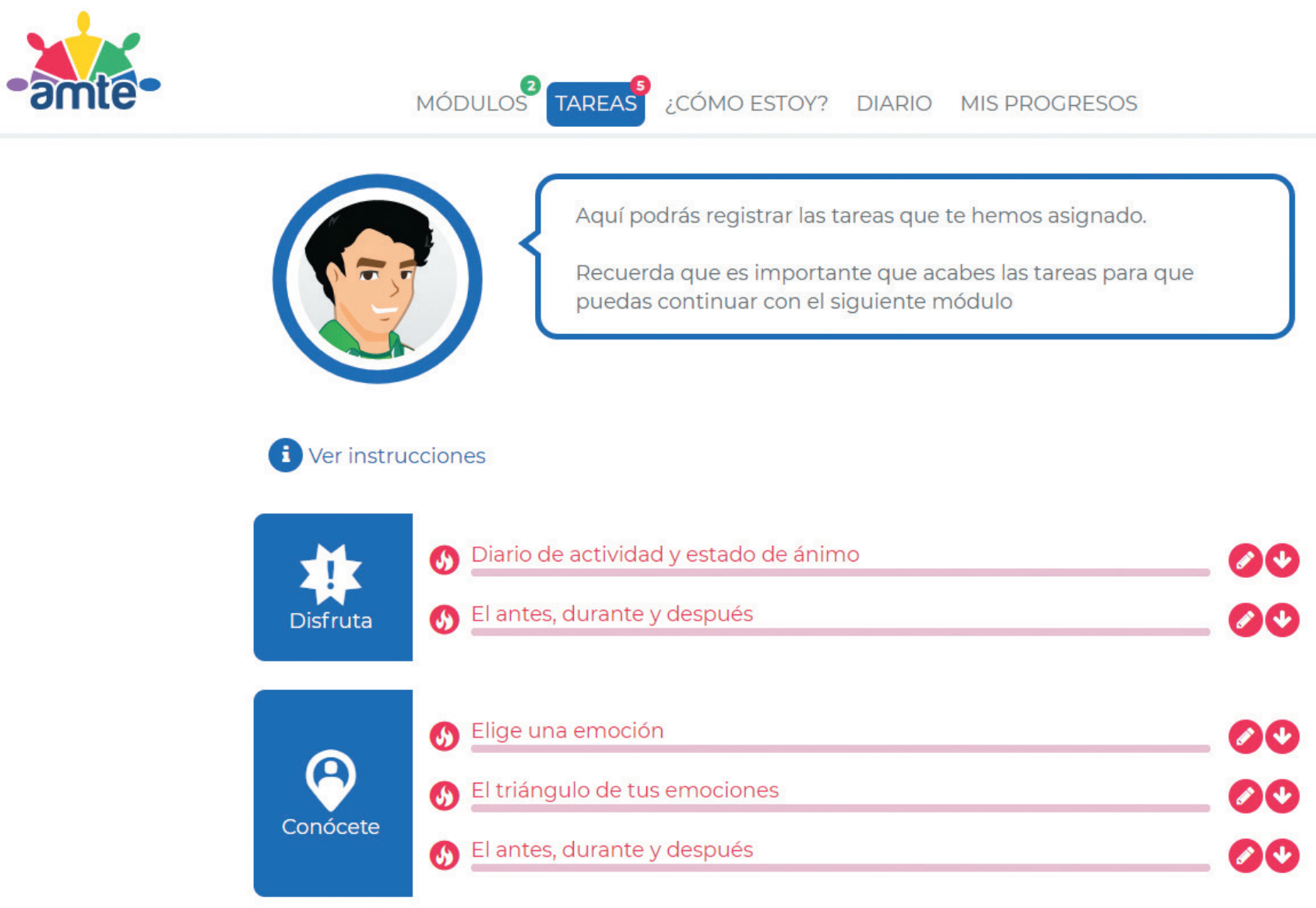

Figura 1. Ejemplos de tareas asignadas al usuario correspondientes a dos de los módulos (en la herramienta Módulos se indica que ha completado los dos primeros módulos). El color actual (en rojo) de las denominaciones de las tareas cambia a medida que se van completando por el usuario. Arriba, en Tareas, se indica que hay 5 tareas sin realizar.

bipolares de ansiedad-relajación y tristeza-alegría. La actividad diaria evalúa el tiempo en que el adolescente ha participado en actividades físicas fuera de las clases de educación física del colegio (correr, bici, jugar al aire libre, etc.). La evaluación se efectúa con la escala EDAAF (véase la sección de Instrumentos de evaluación).

Evaluación semanal. Mediante la herramienta de evaluación semanal se estiman los niveles de ansiedad, depresión, emociones positivas, y actividades (actividad física y actividades de ocio). Transcurrida una semana trabajando con el módulo (generalmente al final del módulo), esta herramienta lleva al usuario a estimar su nivel de ansiedad, depresión, emociones positivas y actividades correspondientes a la última semana, el cual podrá ser consultado durante la semana (véase la Figura 3). La evaluación se lleva a cabo con los siguientes instrumentos: OASISN, ODSISN, MEP, MAF, y MAO (véase la sección de Instrumentos de evaluación).

Mis progresos. A través de esta herramienta el usuario puede ver la evolución de sus síntomas y actividades durante el proceso del tratamiento. Puede chequear en cualquier momento sus progresos, y también puede comprobar que la actividad física y la realización de actividades agradables se asocian a mejoras en las variables de ansiedad y del estado de ánimo. Las variables que se registran en esta herramienta son las que corresponden a las evaluaciones diarias y semanales.

\section{Portal padres}

Es un portal diseñado para que los padres puedan participar como apoyo en el tratamiento de su hijo/a. A través de este portal se proporciona información a los padres sobre las características y funcionamiento del programa, y estos pueden hacer un seguimiento general sobre el uso de la aplicación, incluyendo el trabajo con los módulos y el uso del sistema. También se instruye para que los padres apoyen y motiven a su hijo/a en el uso de la aplicación y la realización de actividades prácticas. 

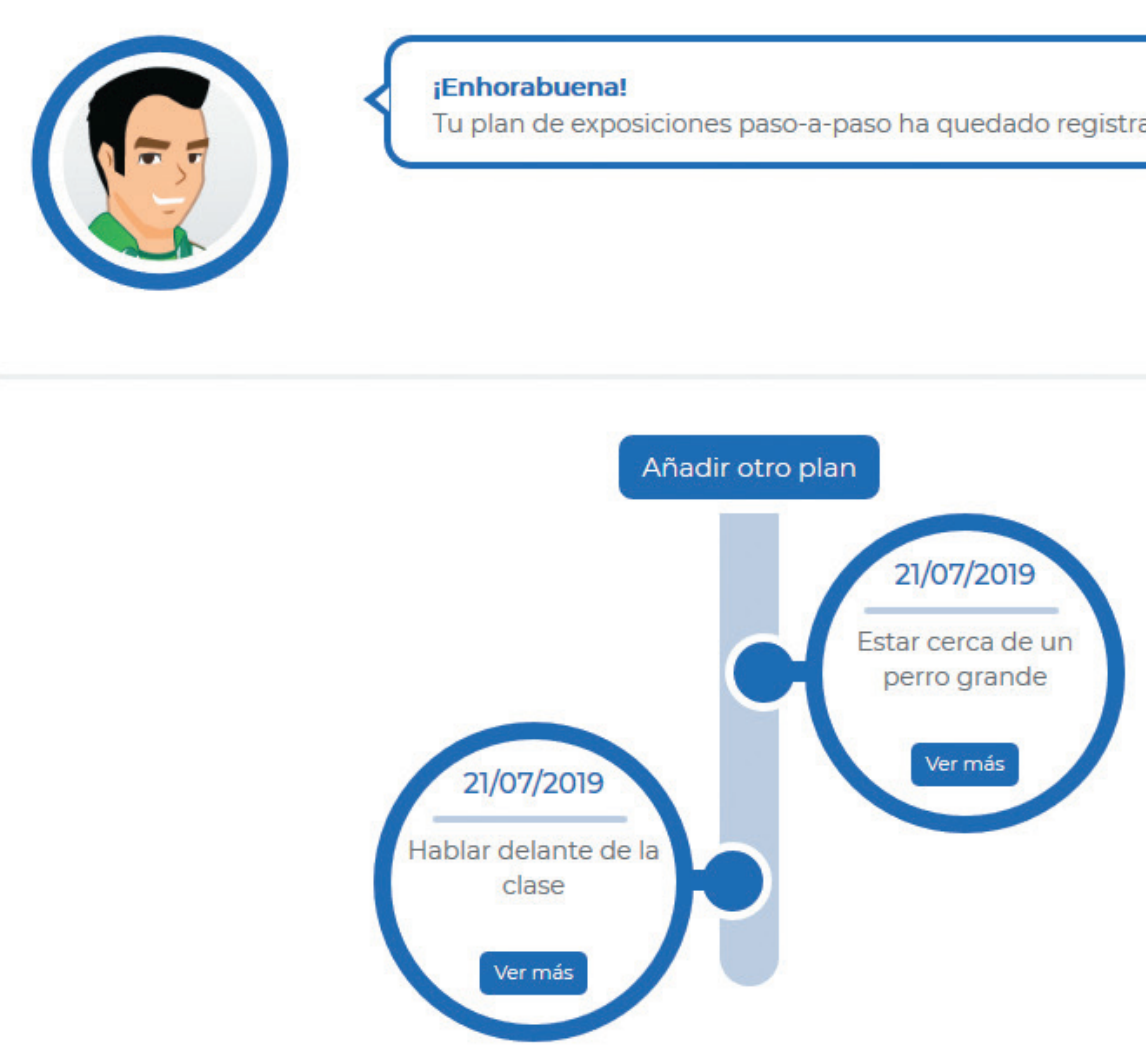

$\leftarrow$ Volver al listado de tareas

Figura 2. Ejemplos de registros de dos prácticas de la tarea «Plan de exposiciones paso-a-paso» (un registro se refiere a un plan de exposición para hablar delante de la clase, y otro para estar cerca de un perro grande). Tras clicar en cada registro ("Ver más) en la aplicación puede consultarse el desarrollo de la práctica realizada.

\section{Portal terapeuta}

El terapeuta tiene acceso al trabajo y progresión del usuario a través de este portal. De este modo, el terapeuta puede tener información general sobre el paciente, sobre el estado de los módulos, y sobre el estado de cumplimentación de las tareas.

\section{Método}

\section{Diseño}

El presente estudio consiste en un ensayo controlado aleatorizado con una muestra de 80 participantes. Estos son asignados al azar a una de las dos condicio- nes del estudio, i.e., condición de aplicación del protocolo iUP-A (grupo experimental) y condición de lista de espera (grupo de control). A los participantes del grupo de control se les ofrecerá la posibilidad de recibir posteriormente el protocolo de tratamiento. Las evaluaciones se efectuarán tras la aleatorización y asignación al grupo correspondiente (pre-tratamiento), después del tratamiento (post-tratamiento), y durante el seguimiento (a los 3-, 6- y 12 meses después del tratamiento). El estudio se llevará a cabo de acuerdo con las directrices indicadas por Consolidated Standards of Reporting Trials (CONSORT; http://www.consort-statement.org/). En la Figura 1 se indica el diagrama de flujo del estudio. 


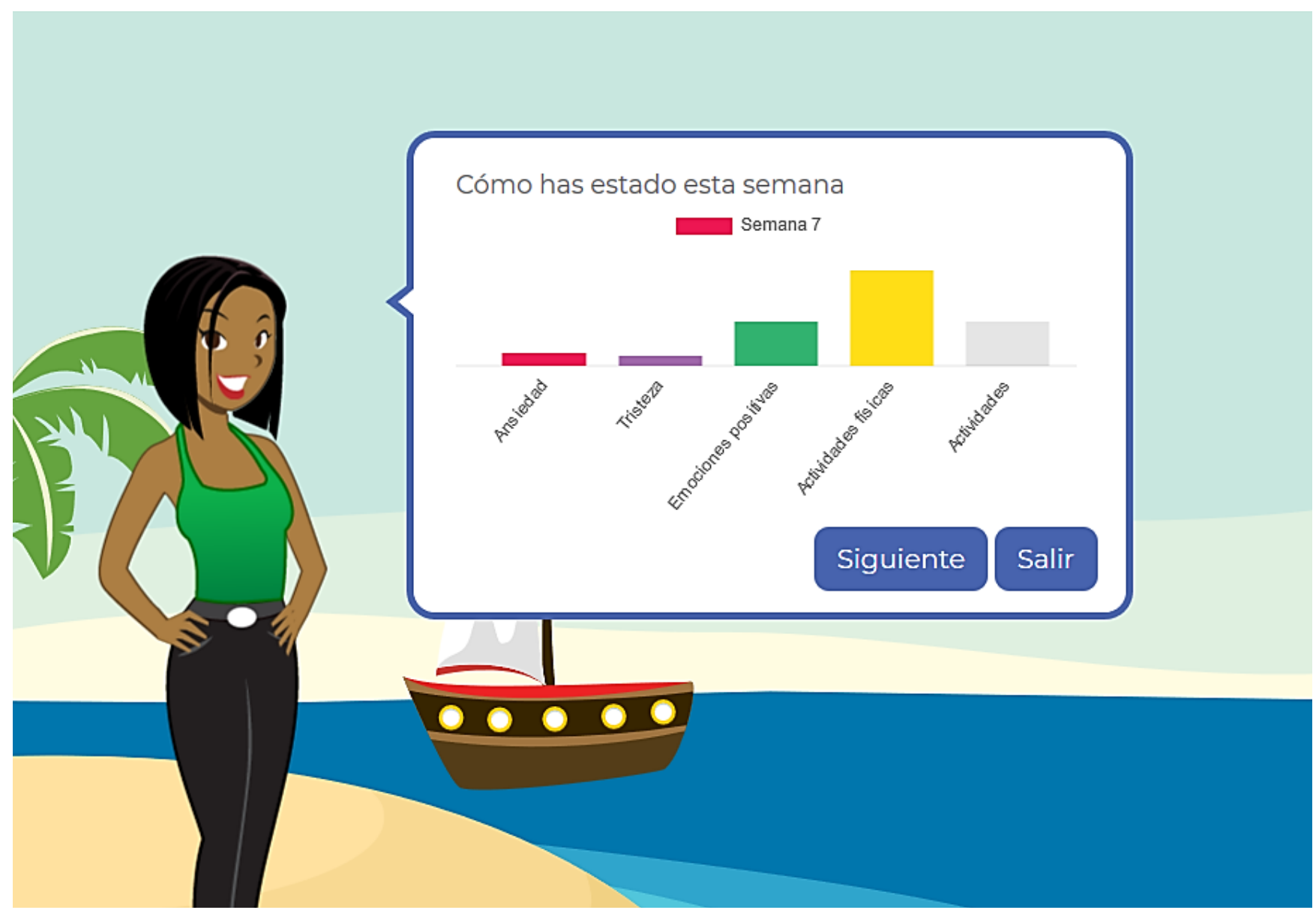

Figura 3. Ejemplo de registro correspondiente a la semana 7 de participación en el programa. El registro refleja las puntuaciones en las variables de evaluación semanal (i.e., ansiedad, depresión, emociones positivas, actividad física y actividades agradables). El adolescente pude apreciar que sus niveles de ansiedad y tristeza son bajos comparados con las puntuaciones en las restantes variables (variables positivas), lo cual se debe a los efectos favorables de la intervención.

\section{Tamaño de la muestra}

Para la estimación del tamaño de la muestra se adoptó un criterio conservador, asumiendo un tamaño del efecto de 0.50 ( $d$ de Cohen). Este es un tamaño del efecto medio de diferencias entre el grupo de intervención activa y el grupo de control (a mayor tamaño del efecto se precisa menor tamaño de la muestra para obtener diferencias significativas). Para la determinación del tamaño de la muestra nos basamos en una potencia estadística del $80 \%(\beta=$ $0.20)$, un nivel de significación del $5 \%(\alpha=0.05)$, y un tamaño del efecto de 0.50 . Aplicando el procedimiento sugerido por Chow, Shao y Wang (2008) para la determinación del tamaño muestral en ensayos clínicos paralelos de dos grupos, se estimó en 64 el número necesario de participantes. Aunque no existen datos fiables sobre la proporción de abandonos en TCC aplicada a través de internet en adolescentes (Vigerland et al., 2016), algunos estudios recientes sugieren que esta es baja (inferior al
10\%; Bonnert et al., 2019). Asumiendo un 20\% de abandonos, el tamaño final de la muestra fue establecido en 80 participantes (40 participantes por cada grupo).

\section{Criterios de inclusión y exclusión}

En el estudio se incluyen participantes que reúnan los siguientes criterios (criterios de inclusión): (a) poseer ente 12 y 17 años de edad; (b) capacidad para comprender y hablar en español; (d) disponer de acceso a internet, correo electrónico, y ordenador personal o tableta electrónica; y (e) reunir los criterios para el diagnóstico de más de un trastorno emocional (i.e., trastornos de ansiedad y/o depresivos) o presentar niveles elevados (nivel clínico o subclínico) de síntomas relacionados con estos trastornos.

Los criterios de exclusión establecidos fueron los siguientes: (a) tener alguna condición psicológica grave, tales como discapacidad intelectual, retraso grave del aprendizaje o trastorno del espectro del autismo, autole- 


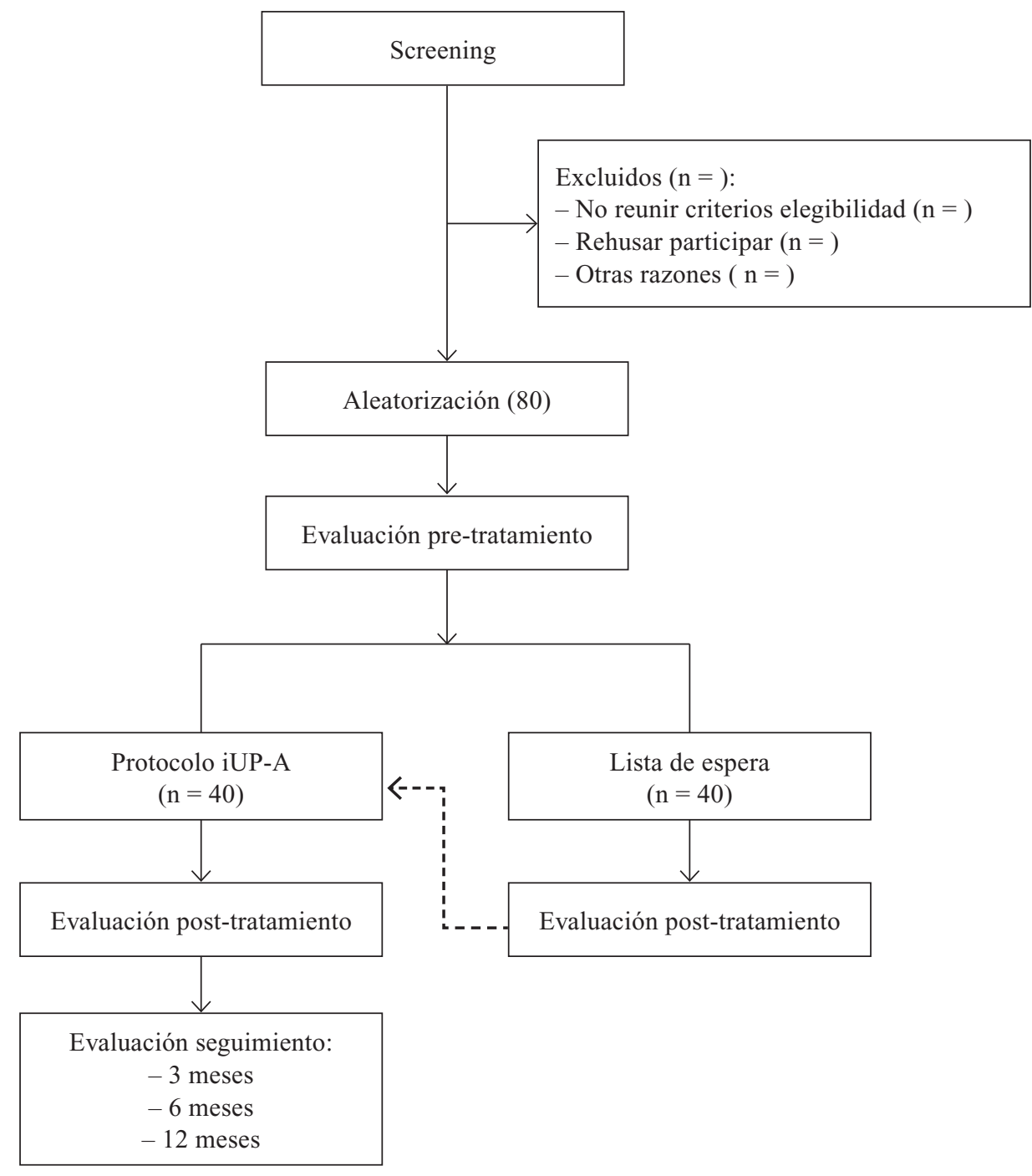

Figura 4. Diagrama de flujo del estudio

siones o ideación suicida, trastorno psicótico, trastorno bipolar o trastorno depresivo grave, y adicción a sustancias; (b) tomar medicación para tratar algún problema psicológico o psiquiátrico con dosis no estables en los últimos 3 meses (un incremento de la medicación durante el curso del estudio también sería motivo de exclusión); (c) estar bajo algún tipo de tratamiento psicológico; y (d) tener alguna enfermedad médica grave incompatible con los requerimientos básicos de la participación en el programa de tratamiento.

\section{Aspectos éticos}

El estudio se lleva a cabo de acuerdo con las directrices de la Declaración de Helsinki, la normativa actual de la Unión Europea y la legislación vigente española sobre protección de pacientes en los ensayos clínicos. Todos los sujetos participan de forma voluntaria en el estudio. Si el participante tiene menos de 16 años, los padres firman el correspondiente consentimiento informado. No obstante, es firmado por el propio adolescente si este tiene 16 años o más. En cualquier caso, el consentimiento se firma tras haber sido explicado suficientemente el estudio tanto al adolescente como a los padres. Siguiendo la normativa sobre protección de datos, estos serán tratados en ficheros protegidos e independientes, estando separado el fichero de información sobre el paciente (edad, sexo, etc.) del fichero (anonimizado) de datos obtenidos en el estudio. Sólo los investigadores responsables podrán acceder a dichos ficheros, garantizándose de esta forma la privacidad de la información. El estudio fue aprobado el Comité de Bioética de la Universidad Nacional de Educación a Distancia (Madrid, España). Así mismo, el ensayo está registrado en ClinicalTrials.gob 
con el identificador NCT04182061. El estudio se encuentra actualmente en fase activa y en proceso de incorporación de participantes.

\section{Participantes y procedimiento}

Los participantes son adolescentes residentes en cualquier provincia española incorporados al estudio a partir de información transmitida por diversas vías, incluyendo internet, centros de salud mental y colegios. La información se refiere a las características y estructura del tratamiento, los criterios de inclusión/exclusión y las vías y datos de contacto con los investigadores del estudio. Inicialmente la invitación para participar en el estudio se lleva a cabo a través de los padres del adolescente. Uno de los investigadores contacta telefónicamente con la persona interesada en participar (generalmente uno de los padres del adolescente) y, tras informarle de forma más detallada sobre el programa de intervención, realiza el screening para determinar si el adolescente cumple los criterios de elegibilidad (criterios de inclusión/exclusión). Si el adolescente pasa este primer filtro, se da de alta en la aplicación tanto a los padres como al adolescente (en cuentas separadas) y se firma el consentimiento informado según proceda. Posteriormente un miembro del equipo investigador aplica la entrevista estructurada MINI-KID versión DSM-IV adaptada a adolescentes (Colón-Soto, Díaz, Soto y Santana, 2005) para establecer el diagnóstico clínico y asegurarse de que el participante cumple, a este respecto, los criterios de inclusión. Una vez admitido y registrado en el estudio, el participante es asignado aleatoriamente al grupo de intervención o al grupo de lista de espera; el proceso se lleva a cabo mediante una secuencia de asignación generada con el ordenador (véase la Figura 4). La participación en el estudio es voluntaria y el sujeto podrá retirarse del mismo en cualquier momento sin exigir ningún tipo de explicación ni prestación. Todos los miembros del equipo investigador son psicólogos clínicos o psicólogos sanitarios, con amplia experiencia en psicopatología, diagnóstico y tratamiento de los trastornos emocionales en niños y adolescentes.

\section{Intervención}

La intervención consiste en una adaptación del Unified protocol for transdiagnostic treatment of emotional disorders in adolescents (UP-A; Ehrenreich-May et al., 2018) para ser aplicado a través de internet (iUP-A) a adolescentes de habla española. La implementación del protocolo iUP-A se llevó a cabo a través de la aplicación web Aprende a Manejar tus Emociones (AMTE). El tratamiento se efectúa a través de esta aplicación. AMTE consiste en un programa web autoaplicable de TCC basada en la evidencia. Es un programa estructurado, paso-a-paso, que el individuo puede seguir a su propio ritmo y según su propia conveniencia (véase atrás).

De modo similar al UP-A, el iUP-A es un tratamiento cognitivo-conductual transdiagnóstico dirigido a modificar los procesos o mecanismos psicopatológicos comunes a los trastornos emocionales, incluyendo la afectividad negativa, la evitación emocional, la rigidez cognitiva, la resolución de problemas, los comportamientos desadaptativos impulsados por emociones, etc. Los principales objetivos del iUP-A consisten en: (1) reducir el malestar emocional (distrés) asociado a emociones perturbadoras e incrementar la emociones positivas, (2) conocer los mecanismos de la evitación emocional y reducir el círculo vicioso de la evitación, (3) potenciar la flexibilidad cognitiva, (4) proporcionar recursos de resolución de problemas, (5) aprender a afrontar adaptativamente las emociones interoceptivas (emociones asociadas a las sensaciones corporales) y exteroceptivas (situaciones emocionales, o emociones desencadenadas por agentes externos), y (5) en términos generales aprender a regular las emociones de forma adaptativa.

La duración del programa es de 8 semanas (el participante tiene acceso a la plataforma durante este tiempo). Como norma se recomienda trabajar con cada módulo durante una semana (hasta pasada una semana no se activa el siguiente módulo). Durante el periodo de intervención, la aplicación envía periódicamente al participante recordatorios y mensajes de refuerzo a través de correo electrónico relacionados con diferentes actividades (seguimiento del módulo, ejercicios, tareas, etc.). Sin dejar de ser rigurosa, la aplicación pretende ser atractiva y ajustarse al rango de edad de los participantes. Por ejemplo, justo al comenzar a trabajar con el programa el adolescente debe configurar el aspecto de su propio avatar (género, apariencia, vestimenta, etc.), el cual le acompañará durante todo el tratamiento. La aplicación cuenta también con un avatar «doctor». Ambos avatares (el configurado por el adolescente y el «doctor») tienen la función de ayudar al paciente durante el proceso de la intervención, reforzando su trabajo, informando sobre los ejercicios y tareas, enfatizando aspectos importantes, etc. Un investigador contacta semanalmente por teléfono durante aproximadamente $10 \mathrm{mi}$ nutos, tanto con el participante como con los padres, para resolver cualquier duda o dificultad relacionada con el uso de la plataforma y la cumplimentación de las tareas, así como también para sugerir y/o animar sobre cualquier cuestión relevante. 
Tabla 2. Instrumentos de evaluación del estudio, con indicación de sus objetivos de evaluación y el periodo en que se aplican

\begin{tabular}{|c|c|c|}
\hline Instrumento & Faceta evaluada & Periodo de aplicación \\
\hline MINI-KID & Diagnóstico clínico & Antes del tratamiento \\
\hline RCADS-30 & Síntomas trastornos de ansiedad y depresivos & Pre-/Post-tratamiento y seguimiento \\
\hline EAN & Ansiedad general & Pre-/Post-tratamiento y seguimiento \\
\hline $\mathrm{CDN}$ & Depresión & Pre-/Post-tratamiento y seguimiento \\
\hline PANASN & Afecto positivo y negativo & Pre-/Post-tratamiento y seguimiento \\
\hline CASI & Sensibilidad a la ansiedad & Pre-/Post-tratamiento y seguimiento \\
\hline EASI-A & Evitación emocional & Pre-/Post-tratamiento y seguimiento \\
\hline SWLSN & Satisfacción con la vida & Pre-/Post-tratamiento y seguimiento \\
\hline PSWQN-11 & Preocupación ansiosa & Pre-/Post-tratamiento y seguimiento \\
\hline SASC-R & Ansiedad social & Pre-/Post-tratamiento y seguimiento \\
\hline PDSS-SR & Gravedad trastorno de pánico & Pre-/Post-tratamiento y seguimiento \\
\hline EDAAF & Ansiedad/depresión y actividad física & Durante el tratamiento \\
\hline OASISN & Ansiedad & Durante el tratamiento \\
\hline ODSISN & Depresión & Durante el tratamiento \\
\hline MEP & Estado de ánimo positivo & Durante el tratamiento \\
\hline MAF & Actividad física & Durante el tratamiento \\
\hline MAO & Actividades de ocio & Durante el tratamiento \\
\hline CSP & Satisfacción con el programa & Después del tratamiento \\
\hline
\end{tabular}

Nota: MINI-KID = MINI Neuopsychiatric Diagnostic Interview para Niños y Adolescentes; EAN = Escala de Anxiedad para Niños y Adolescentes; $\mathrm{CDN}=$ Cuestionario de Depresión para Niños y Adolescentes; PANASN = Cuestionario PANAS para Niños y Adolescentes; CASI = Childhood Anxiety Sensitivity Index; EASI-A = Emotional Avoidance Strategy Inventory for Adolescents; SWLSN = Escala de Satisfacción con la Vida para Niños y Adolescentes; PSWQN-11 = Cuestionario PSWQ-11 para Niños y Adolescentes; SASC-R = Social Anxiety Scale for Children-Revised; PDSS-SR = Panic Disorder Severity Scale-Self-Report; CSP = Cuestionario de Satisfacción con el Programa; EDAAF = Evaluación Diaria del Ánimo y la Actividad Física; OASISN = Overall Anxiety Severity and Impairment Scale para Niños y Adolescentes; ODSISN = Overall Depression Severity and Impairment Scale para Niños y Adolescentes; MEP = Mis Emociones Positivas; MAF = Mi Actividad Física; MAO = Mis Actividades de Ocio; CSP = Cuestionario de Satisfacción con el Programa.

\section{Instrumentos de evaluación}

A continuación se indican los instrumentos de evaluación utilizados en el estudio. En la Tabla 2 se especifica el tipo de constructo evaluado y los momentos temporales en que se aplica.

\section{Entrevista diagnóstica}

Mini International Neuropsychiatric Interview for Children and Adolescents (MINI-KID, versión 1.1; Sheehan, Lecrubier,Shytle, Milo, y Hergueta, 2000), versión española de Colón-Soto et al., 2005). Entrevista de diagnóstico clínico estructurada, adaptada para niños y adolescentes. Posibilita el diagnóstico de los principales trastornos mentales en un periodo de tiempo relativamente breve, según los criterios diagnósticos del DSM-IV y de la CIE-10. Los autores han referido niveles aceptables de validez y fiabilidad, con valores de concordancia diagnóstica entre jueces (coeficiente kappa) superiores a .80 .

\section{Medidas de resultado primarias}

Revised Child Anxiety and Depression Scale-30 (RCADS-30; Sandín, Chorot, Valiente y Chorpita, 2010). Es una escala de autoinforme de 30 ítems ampliamente utilizada para evaluar los síntomas de los trastornos de ansiedad y depresivos en niños y adolescentes. Incluye las seis subescalas siguientes basadas en los síntomas criteriales descritos en el DSM-IV: trastorno depresivo mayor, trastorno de pánico, fobia social (trastorno de ansiedad social), trastorno de ansiedad de separación, trastorno de ansiedad generalizada, y trastorno obsesivo-compulsivo. Cada ítem se responde con una estimación de frecuencia entre 0 («Nunca») y 3 («Siempre»), indicando mayor gravedad las puntuacio- 
nes más altas. Puede obtenerse una puntuación para cada subescala por separado, una puntuación total, y una puntuación global para los síntomas de los trastornos de ansiedad. La RCADS-30 ha demostrado buenas propiedades psicométricas tanto con población no clínica como clínica (Sandín et al., 2010; Piqueras, Martín-Vivar, Sandín, San Luis y Pineda, 2017; Piqueras, Pineda, Martín-Vivar y Sandín, 2017), con valores de consistencia interna ( $\alpha$ de Cronbach) que varían entre .66 y .86 para las subescalas, entre .86 y .88 para la escala total, y entre .82 y .90 para la escala general de ansiedad.

Escala de Ansiedad para Niños (EAN; Sandín, Chorot y Valiente, 2016). La EAN es una escala de autoinforme de 10 ítems diseñada para efectuar una evaluación rápida y no contaminada de la ansiedad en niños y adolescentes. Evalúa los principales síntomas de ansiedad teniendo en cuenta las diferentes facetas de la misma (fisiológica, cognitiva, social, etc.). La persona contesta indicando la frecuencia con que ha experimentado los síntomas de ansiedad según una escala de respuesta de 4 puntos que varía entre 0 («Nunca o casi nunca») y 3 («Muchas veces o casi siempre»). Se obtiene una puntuación total en ansiedad sumando los 10 ítems de la escala. Se han indicado valores elevados de consistencia interna de la escala ( $\alpha$ entre .92 y .95) (Sandín et al., 2016).

Cuestionario de Depresión para Niños y Adolescentes (CDN; Sandín et al., 2016). Consiste en una escala de autoinforme de 16 ítems elaborada para evaluar de forma rápida y no contaminada el nivel de depresión en niños y adolescentes. La escala cubre los principales síntomas relacionados con el diagnóstico del trastorno depresivo mayor y la distimia (criterios DSM-IV). La persona contesta a cada ítem estimando la frecuencia con que ha experimentado lo indicado en cada ítem según una escala de 3 puntos que varía entre 0 («Nunca o casi nunca») y 2 («Muchas veces o casi siempre»). Se ha constatado que la CDN posee elevada consistencia interna $(\alpha=.91)$ (Sandín, Valiente, Sánchez-Arribas, y Chorot, 2013).

\section{Medidas de resultado transdiagnósticas}

Cuestionario PANAS para Niños y Adolescentes (PANASN; Sandín, 2003). Cuestionario de autoinforme diseñado para evaluar el afecto positivo y negativo en niños y adolescentes. Consta de 20 ítems (10 evalúan el afecto positivo y 10 el afecto negativo), las cuales se contestan según una escala de frecuencia de 3 puntos que varía entre 1 («Nunca») y 3 («Muchas veces»), indicando hasta qué punto se ha experimentado cada emoción. El cuestionario posee adecuadas propiedades psi- cométricas, con valores de consistencia interna que varían entre .72 y .82 para el afecto positivo, y entre .74 y .81 para el afecto negativo (Molina, Sandín y Chorot, 2014; Sandín, 2003).

Childhood Anxiety Sensitivity Index (CASI; Silverman, Fleisig, Rabian y Peterson, 1991); versión española de Sandín (1997; Sandín, Chorot, Santed y Valiente, 2002). La CASI es una escala de autoinforme de 18 ítems diseñada para evaluar, en niños y adolescentes, la sensibilidad a la ansiedad, o reacciones de malestar a los síntomas de ansiedad. El participante estima la frecuencia con la que experimenta lo indicado en cada ítem según una escala de respuesta de 3 puntos, variando entre 1 («Nunca») y 3 («Muchas veces»). La versión española ha demostrado poseer adecuadas propiedades psicométricas, con niveles elevados de consistencia interna (valores de $\alpha$ entre .80 y .89) (Sandín et al., 2002; Valiente, Sandín y Chorot, 2002).

Emotional Avoidance Strategy Inventory for Adolescents (EASI-A; Kennedy y Ehrenreich-May, 2016); versión española de García-Escalera, Chorot, Valiente, Sandín et al., 2016. Escala de autoinforme de 17 ítems en la que la persona debe contestar estimando hasta qué punto es verdadero lo indicado en cada ítem, según una escala de respuesta de 5 puntos que varía entre 0 («Nunca o casi nunca») y 4 («Siempre o casi siempre»). Se ha sugerido que posee propiedades psicométricas adecuadas y correlaciones positivas con ansiedad y depresión (Kenney y Ehrenreich-May, 2016). La consistencia interna de la versión española es elevada, con valores del coeficiente $\alpha$ de Cronbach entre 86 y .87 (García-Escalera et al., 2017).

\section{Medidas de resultado secundarias}

Escala de Satisfacción con la Vida para Niños y Adolescentes (SWLSN; Sandín, Chorot y Valiente, 2012). Se trata de una escala de autoinforme de 5 ítems diseñada para evaluar el grado en que el individuo está satisfecho con su vida. Es una versión adaptada a la población de niños y adolescentes a partir de la escala para adultos Satisfaction with Life Scale (SWLS; Diener, Emmons, Larsen y Griffin, 1985). La persona responde indicando el grado en que se asemeja a su vida lo indicado en cada ítem, con un rango de respuesta de 4 puntos variando entre 1 («No en absoluto») y 4 («Mucho o totalmente»). La consistencia interna de la escala es buena, habiéndose obtenido valores de $\alpha$ entre .81 y .89 (García-Escalera et al., 2017).

Cuestionario PSWQ-11 para Niños y Adolescentes (PSWQN-11; Sandín, Chorot y Valiente, 2010). Es una adaptación para niños y adolescentes de la versión de 11 
ítems del Penn State Worry Questionnaire (Sandín, Chorot, Valiente y Lostao, 2009). Los 11 ítems se refieren a la preocupación ansiosa y se estiman indicando la respuesta que mejor se ajuste a la forma de sentir de la persona según una escala de respuesta de 5 puntos, variando entre 1 («Nada de acuerdo») y 5 («Totalmente de acuerdo»). Tanto la consistencia interna $(\alpha=.92)$ como la estabilidad temporal un mes (test-retest $r=.85$ ) del PSWQ11 son excelentes (Sandín et al., 2009).

Social Anxiety Scale for Children-Revised, SASC-R (La Greca y Stone, 1993); versión española de Sandín (1997). Es una escala multidimensional de la ansiedad que consta de 18 ítems. La persona contesta indicando la frecuencia con la que experimenta cada síntoma de ansiedad social según una escala de respuesta de 3 puntos, variando las estimaciones entre 1 («Nunca») y (3) («Muchas veces»). Permite obtener una medida global de ansiedad social sumando las puntuaciones de cada ítem. Datos psicométricos sobre la estructura factorial, la fiabilidad y la validez de la versión española sugieren que la escala posee buenas propiedades psicométricas, con excelente nivel de consistencia interna $(\alpha=.90)$ (Sandín, Chorot, Valiente, Santed y Sánchez-Arribas, 1999; Sandín, Valiente, Chorot, Santed y Sánchez-Arribas, 1999).

Panic Disorder Severity Scale-Self-Report (PDSSSR; Houck et al., 2002). Es una versión de autoinforme de la clásica Panic Disorder Severity Scale (Shear et al. (1997), una escala de estimación clínica diseñada para evaluar la gravedad del trastorno de pánico. Aplicamos la versión española adaptada por Sandín (2010). Consiste en 7 ítems, estimados según una escala ordinal de gravedad de 0 a 4 (rango de puntuación total $=0$-28). Los ítems se refieren a la última semana y evalúan la frecuencia de los ataques de pánico, el nivel de malestar asociado a los ataques de pánico, preocupación o nerviosismo por nuevos ataques, evitación exteroceptiva, evitación interoceptiva, interferencia laboral y familiar, e interferencia social. En la validación al español de la escala se obtuvo un coeficiente $\alpha$ de .93 (Sánchez-Arribas, Chorot, Valiente y Sandín, 2015).

Evaluación de la experiencia de usuario. Se evaluará hasta qué punto la aplicación AMTE ha resultado ser: (a) viable (factibilidad) y se ajusta convenientemente a la rutina del usuario, (b) fácilmente utilizable (usabilidad) y sin especiales complicaciones, y (c) una experiencia agradable (aceptabilidad). Para evaluar las dos primeras variables se pregunta al usuario sobre ambos aspectos al final del tratamiento (también se computará el uso del sistema). Para evaluar la aceptabilidad se aplicará el Cuestionario de Satisfacción con el Programa (CSP). Consiste en un cuestionario de 4 ítems diseñado para evaluar la aceptabilidad del programa. Se basa en el cuestionario sobre satisfacción con el tratamiento publicado por Rapee et al. (2006), e incluye cuatro preguntas relacionadas con la percepción sobre las habilidades adquiridas para manejar las emociones, la eficacia del programa, lo agradable o atractivo del programa, y la disponibilidad a recomendarlo a otros chicos/as. Cada pregunta se evalúa según una escala de 0 («Poco o nada») a 10 («Mucho»). El cuestionario se aplica al final del tratamiento.

\section{Medidas intra-tratamiento}

Evaluación Diaria del Ánimo y la Actividad Física (EDAAF). Se trata de una escala que se aplica diariamente durante el periodo de tratamiento (plataforma AMTE). Evalúa las dimensiones de ansiedad/relajación y tristeza/ alegría, en un rango de 6 puntos. También evalúa los días en los que se haya practicado alguna actividad física durante el tiempo de ocio (i.e., no relacionada con clases de educación física en el colegio); se puntúa según un rango de 6 puntos, variando entre 0 (si no se ha dedicado ningún tiempo) y 6 (se ha dedicado más de una hora).

Overall Anxiety Severity and Impairment Scale (OASIS; Norman, Cissell, Means-Christensen y Stein, 2006). Versión en español adaptada para ser aplicada a través de internet (plataforma AMTE) a población de niños y adolescentes (OASISN). Consta de 5 ítems referidos a la ansiedad experimentada durante la última semana, incluyendo los niveles de frecuencia, intensidad y evitación, y el grado de interferencia familiar, académica y social. La estimación se lleva a cabo sobre la base de una escala de medida de 5 puntos, que varía entre 0 («Nunca»/«Nada») y 4 («Siempre»/«Muchísimo»). Los autores de la versión original para población adulta (Norman et al., 2006) han proporcionado evidencia sobre consistencia interna, fiabilidad y validez.

Overall Depression Severity and Impairment Scale (ODSIS; Bentley, Gallagher, Carl, y Barlow, 2014). Versión en español adaptada para ser aplicada a través de internet (plataforma AMTE) a población de niños y adolescentes (ODSISN). Consta de 5 ítems referidos a la depresión y anhedonia experimentadas durante la última semana, incluyendo los niveles de frecuencia e intensidad, y el grado de interferencia familiar, académica y social. La estimación se lleva a cabo según una escala de medida de 5 puntos, que varía entre 0 («Nunca»/«Nada») y 4 («Siempre»/«Muchísimo»). Los autores de la versión original para población adulta (Bentley et al., 2014) han proporcionado evidencia sobre consistencia interna, fiabilidad y validez.

Mis Emociones Positivas (MEP). Escala diseñada para evaluar semanalmente el estado de ánimo positivo. 
Incluye dos ítems relacionados, respectivamente, con la frecuencia e intensidad del estado emocional positivo experimentado durante la última semana. La persona responde a cada ítem según una escala de medida de 5 puntos, que varía entre $0(«$ Nunca $» / \ll$ Nada $»)$ y 4 («Siempre»/«Muchísimo»).

Mi Actividad Física (MAF). Escala diseñada para evaluar semanalmente el nivel de actividad física. Evalúa mediante un ítem el número de días en los que se ha llevado a cabo algún tipo de actividad física recreativa (no académica) durante al menos una hora, a lo largo de la semana. El rango de puntuación varía entre 0 (ningún día) y 7 (los siete días).

Mis Actividades de Ocio (MAO). Escala diseñada para evaluar semanalmente la práctica de actividades recreativas. Posibilita, mediante 4 ítems, la evaluación de la participación del adolescente en cada uno de los siguientes tipos de actividades de ocio: actividades de atención o voluntariado, actividades sociales agradables, actividades agradables realizadas individualmente, y actividades formativas. El chico/a estima el número de días en los que llevó a cabo cada tipo de actividad a lo largo de la semana. El rango de puntuación para cada ítem varía entre 0 (ningún día) y 7 (los siete días).

\section{Análisis estadísticos}

Los análisis de los datos se llevarán a cabo teniendo en cuenta el principio de «intención de tratar» (intention-to-treat), así como también la participación en la totalidad del protocolo (per-protocol analysis). Con objeto de verificar la validez de la aleatorización, i.e., que no existan diferencias entre ambos grupos en la situación basal (situación pre-tratamiento), los grupos serán comparados en todas las variables, incluidas las variables sociodemográficas. Para ello se aplicará la prueba de $t$ de Student (para las variables continuas) o la prueba de tablas cruzadas (para las variables categóricas). Los abandonos (attrition) se analizarán mediante análisis de regresión logística para examinar los perfiles de pérdidas durante el tratamiento y post-tratamiento.

Los cambios intra e intergrupo serán calculados a través de la estimación del tamaño del efecto, computándose para ello el tamaño del efecto estandarizado $d$ de Cohen (diferencia entre medias estandarizada; Cohen, 1977), o la forma modificada por Hedges (1981; $g$ de Hedges) en casos de muestras pequeñas $(<20)$. En la interpretación del tamaño del efecto seguiremos los estándares aceptados actualmente, los cuales sugieren que 0.50 y 0.20 corresponden a tamaños del efecto medio y bajo, respectivamente, siendo tamaños del efecto altos a partir de 0.80 .
Se examinarán los efectos del tratamiento (iUP-A vs. lista de espera) sobre los distintos tipos de variables durante el post-tratamiento y el seguimiento. Para ello se aplicarán ecuaciones de estimación generalizadas (generalized stimating equations, GEE). Este modelo de estimación es apropiado para examinar los cambios en las «medidas de resultado» (outcome measures) en el tiempo (p.ej., cambios en la sintomatología). Este tipo de análisis posee ciertas ventajas sobre otros métodos estadísticos clásicos para estimar los efectos temporales de una variable, incluyendo el control del análisis de la intención de tratar, una menor pérdida de potencia estadística en los estudios que utilizan muestra pequeñas y elevado número de medidas repetidas, modelar directamente los cambios temporales en los grupos, y calcular el factor de cambio $\exp (\beta)$ (odds ratio, o porcentaje de cambio respecto a la situación basal). Se utilizará el método de estimación de máxima verosimilitud, con una estimación robusta del error.

Con objeto de examinar las trayectorias en el tiempo de grupos de medidas de resultado (p.ej., en las variables de ansiedad y depresión), se aplicarán modelos de curva de crecimiento latente (latent growth curve models, LGCM) referidos al curso del tratamiento y al periodo de seguimiento. En estos modelos, el cambio es modelado en función del tiempo y se representa a través de la especificación de variables latentes (i.e., variables no observadas sino estimadas) referidas como growth factors. Las variables latentes (la intercepción y la pendiente) se estiman sobre la base de las trayectorias individuales, y proporcionan una estimación en el tiempo sobre la trayectoria promedio y sobre la variación individual a lo largo de la trayectoria. Al consistir en variables latentes se considera que se trata de efectos aleatorios (no efectos fijos) (Felt, Depaoli y Tiemensma, 2017).

\section{Discusión}

El presente estudio de protocolo tiene dos objetivos fundamentales. El primero ha consistido en llevar a cabo el desarrollo de una aplicación web y adaptar el UP-A (Ehrenreich et al., 2018) para ser aplicado a través de internet en población de habla española (i.e., desarrollo de la aplicación AMTE). El segundo consiste en proporcionar evidencia preliminar sobre la eficacia del UP-A aplicándolo en su formato online (iUP-A), a través de la plataforma AMTE, en un ensayo controlado aleatorizado para el tratamiento de los trastornos emocionales en población adolescente. Adicionalmente también se pretende obtener información sobre la viabilidad, usabilidad y aceptabilidad (satisfacción con el programa) por los adolescentes de la aplicación AMTE. 
Para la consecución del primer objetivo ha sido necesario un largo periodo de trabajo llevado a cabo por BS, PC y RMV en el que se ha efectuado la construcción de la aplicación AMTE y la implementación del iUP-A. En su construcción se ha intentado integrar el rigor de un protocolo de terapia cognitivo-conductual basado en la evidencia para el tratamiento de los trastornos emocionales con un formato atractivo y funcional apto para niños/adolescentes entre los 12 y los 17 años de edad. La aplicación posee una estructura para un aprendizaje progresivo de las técnicas y habilidades necesarias para el afrontamiento y regulación de los principales problemas emocionales relacionados con alteraciones de la depresión y las diferentes manifestaciones de la psicopatología de la ansiedad (ansiedad de separación, fobias, ansiedad generalizada, pánico, etc.). La plataforma web incluye tres portales de acceso y trabajo separados, i.e., un portal fundamental destinado al usuario y dos portales complementarios, uno para la participación de los padres y otro para la supervisión que lleva a cabo el terapeuta. El portal del usuario contiene diversos tipos de herramientas funcionales, flexibles y transversales, incluyendo herramientas para: (a) la gestión de cada módulo individual (herramienta Módulos), (b) poner en práctica lo que se va aprendiendo (herramienta de Tareas), (c) tener información sobre actividades pendientes y realizadas (herramienta Diario), (d) realizar las evaluaciones diarias y semanales (herramienta ¿Cómo estoy?), y (e) tener feed-back sobre las mejorías durante el curso del tratamiento (herramienta Mis progresos). Así mismo, AMTE incluye diversos materiales multimedia (vídeos, etc.) con la posibilidad de que el usuario pueda descargar hojas informativas y de trabajo, como material de apoyo para la realización de tareas y ejercicios, y poner en práctica lo aprendido en cada módulo.

Aunque en los últimos años han aparecido algunos protocolos diseñados para el tratamiento transdiagnóstico vía internet de los trastornos emocionales en adultos, tanto en España (González-Robles et al., 2015) como fuera de nuestro país (Titov et al., 2012), que nosotros sepamos, AMTE es la primera plataforma online a nivel internacional para el tratamiento transdiagnóstico de los problemas y trastornos emocionales en niños y adolescentes. Al incluir la implementación del protocolo UP-A para la aplicación de este a través de internet (iUP-A), AMTE incluye también el primer UP-A adaptado en formato online. No conocemos que, en estos momentos, exista a nivel internacional ninguna otra versión del UP-A para ser aplicado a través de internet. El UP-A ha sido recientemente adaptado en español, en formato grupal cara-a-cara, para ser aplicado en un contexto de prevención universal y dirigida (indicada y/o selectiva)
(García-Escalera et al., 2019, 2020), pero aún no había sido adaptado para una aplicación a través de internet.

En lo que concierne al segundo objetivo (actualmente en curso de desarrollo), pretendemos obtener información preliminar sobre la eficacia del iUP-A en el tratamiento de los problemas y trastornos emocionales en niños y adolescentes (entre 12 y 17 años de edad), en un ensayo controlado aleatorizado. La muestra incluye tanto participantes obtenidos por internet a partir de la población general como participantes derivados desde centros de salud mental. A este respecto, es importante señalar que nuestro proyecto, no sólo pretende examinar la eficacia del protocolo sobre las clásicas medidas de resultado primarias (i.e., variables de ansiedad y depresión comunes a los diferentes trastornos emocionales) o secundarias (i.e., variables de ansiedad y depresión específicas a los distintos trastornos emocionales), sino que también se centra en variables transdiagnósticas relevantes (véase Sandín et al., 2012), entre las que se incluyen la sensibilidad a la ansiedad, la evitación emocional, y el afecto negativo. Aunque estas últimas variables pueden mediar o modular el efecto del tratamiento sobre las variables de resultado primarias o secundarias, también deberían ser objetivos directos de los programas de tratamiento transdiagnóstico, ya que constituyen componentes nucleares de este nuevo enfoque. Por otra parte, el protocolo también se dirige a la mejora de factores positivos, tales como el afecto positivo y la satisfacción vital; los factores positivos ya han venido siendo señalados como objetivos relevantes de la terapia transdiagnóstica en protocolos diseñados para población adulta (p.ej., Botella, Mira, García-Palacios y Baños, 2015), pero no en los protocolos destinados a niños y adolescentes. Por tanto, otras fortalezas del estudio tienen que ver con la aplicación de un tratamiento novedoso aplicado a la maximización de factores positivos.

Por tanto, en términos generales el desarrollo del presente estudio de protocolo, incluyendo el desarrollo de la aplicación AMTE junto con la implementación del iUP-A, y el estudio piloto contralado aleatorizado, puede suponer un importante avance en el campo de la psicología clínica relacionado con el tratamiento y prevención (especialmente por dirigirse a población infantojuvenil) de los trastornos emocionales. El nuevo enfoque de TCC-T constituye un importante progreso para abordar psicológicamente los trastornos emocionales, superior a los enfoques de la TCC clásica. Por otra parte, la aplicación de este enfoque a través de internet posibilita eliminar muchas barreras propias de la terapia cara-a-cara que impiden o dificultan el acceso a la terapia cognitivo-conductual empíricamente validada. 
Algunas de las limitaciones potenciales del estudio tienen que ver con las propias características de las intervenciones online, incluyendo la necesidad de acceso a internet y disponer de ordenador personal o tableta electrónica, la limitada adherencia a este tipo de intervención, las actitudes negativas hacia la intervención por internet, etc. Estas dificultades se tratan de minimizar mediante la estimulación de la motivación durante el primer módulo, y a través del apoyo directo del terapeuta (mediante contactos periódicos por teléfono directos con el usuario y, en su caso, mensajes de correo electrónico), refuerzos a través de la propia aplicación, y a través del apoyo mediado por los padres del participante.

\section{Conflictos de intereses}

Los autores declaran que no existen conflictos de intereses

\section{Referencias}

Andersen, P., Toner, P., Bland, M., \& McMillan, D. (2016). Effectiveness of transdiagnostic cognitive behaviour therapy for anxiety and depression in adults: a systematic review and meta-analysis. Behavioural and Cognitive Psychotherapy, 44, 673-690.

Andersson, G. (2016). Internet-delivered psychological treatments. Annual Review of Clinical Psychology, 12, 157-179.

Andersson, G., Titov, N., Dear, B. F., Rozental, A., \& Carlbring, P. (2019). Internet-delivered psychological treatments: from innovation to implementation. World Psychiatry, 18, 20-28.

Barlow, D. H., Farchione, T. J., Fairholme, C. P., Ellard, K. K., Boisseau, C. L., Allen, L. B., ... \& Ehrenreich-May, J. (2011). Unifi ed protocol for transdiagnostic treatment of emotional disorders: Therapist guide. New York, NY, US: Oxford University Press.

Bentley, K. H., Gallagher, M. W., Carl, J. R., \& Barlow, D. H. (2014). Development and validation of the Overall Depression Severity and Impairment Scale. Psychological Assessment, 26, 815-830.

Bilek, E. L., \& Ehrenreich-May, J. (2012). An open trial investigation of a transdiagnostic group treatment for children with anxiety and depressive symptoms. Behavior Therapy, 43(4), 887-897.

Bonnert, M., Olén, O., Lalouni, M., Hedman-Lagerlöf, E., Särnholm, J., Serlachius, E., \& Ljótsson, B. (2019). Internetdelivered exposure-based cognitive-behavioral therapy for adolescents with functional abdominal pain or functional dyspepsia: a feasibility study. Behavior Therapy, 50, 177-188.

Botella, C., Mira, A., Herrero, R., García-Palacios, A., y Baños, R. (2015). Un programa de intervención autoaplicado a través de Internet para el tratamiento de la depresión: Sonreir es divertido. Aloma, 33, 39-48.

Chavira, D. A., \& Stein, M. B. (2005). Childhood social anxiety disorder: from understanding to treatment. Child and Adolescent Psychiatric Clinics, 14, 797-818.
Chow, S-C., Shao, J., \& Wang, H. (2008). Sample size calculations in clinical research. Boca Raton, FL: Chapman \& Hall/CRC.

Clark, D. A. (2009). Cognitive behavior therapy for anxiety and depression: Possibilities and limitations of a transdiagnostic perspective. Cognitive Behavior Therapy, 38, 29-34.

Cohen, J. (1977). Statistical power analysis for the behavioral sciences. Hillsdale: Lawrence Erlbaum Associates.

Colón-Soto, M., Díaz, V., Soto, O., y Santana, C. (2005). Mini International Neuropsychiatric Interview para Niños $y$ Adolescentes (MINI-KID). Versión en español. Tampa: Medical Outcome Systems.

Diener, E., Emmons, R. A., Larsen, R. J., Griffin, S. (1985). The satisfaction with life scale. Journal of Personality Assessment, 49, 71-5.

Ehrenreich-May, J., Bilek, E. L., Queen, A. H., \& Hernandez Rodriguez, J. (2012). A unified protocol for the group treatment of childhood anxiety and depression. Revista de Psicopatología y Psicología Clínica, 17, 219-236.

Ehrenreich-May, J., Kennedy, S. M., Sherman, J. A., Bilek, E. L., Buzzella, B. A., Bennett, S. M., \& Barlow, D. H. (2018). Unified protocols for transdiagnostic treatment of emotional disorders in children and adolescents. Therapist guide. New York, NY: Oxford University Press.

Ehrenreich-May, J., Rosenfield, D., Queen, A. H., Kennedy, S. M., Remmes, C. S., \& Barlow, D. H. (2017). An initial waitlistcontrolled trial of the unified protocol for the treatment of emotional disorders in adolescents. Journal of Anxiety Disorders, 46, 46-55.

Essau, C. A., Conradt, J., \& Petermann, F. (1999). Frequency and comorbidity of social phobia and social fears in adolescents. Behaviour Research and Therapy, 37, 831-843.

Felt, J. M., Depaoli, S., \& Tiemensma, J. (2017). Latent growth curve models for biomarkers of the stress response. Frontiers in Neuroscience, 11, 315.

García-Escalera, J., Chorot, P., Sandín, B., Ehrenreich-May, J., Prieto, A., \& Valiente, R. M. (2019). An open trial applying the unified protocol for transdiagnostic treatment of emotional disorders in adolescents (UP-A) adapted as a school-based prevention program. Child Youth Care Forum, 48, 29-53, 2019.

García-Escalera, J., Chorot, P., Valiente, R. M., Sandín, B., Tonarely, N., y Ehrenreich-May, J. (2016). Versión española de la Emotional Avoidance Strategy Inventory for Adolescents (EASI-A). Madrid: Universidad Nacional de Educación a Distancia (no publicado).

García-Escalera, J., Chorot, P., Valiente, R., Reales, J. M., \& Sandín, B. (2016). Efficacy of transdiagnostic cognitivebehavioral therapy for anxiety and depression in adults, children and adolescents: A meta-analysis. Revista de Psicopatología y Psicología Clínica, 21, 147-175.

García-Escalera, J., Valiente, R. M., Chorot, P., Ehrenreich-May, J., Kennedy, S. M., \& Sandín, B. (2017). The Spanish version of the unified protocol for transdiagnostic treatment of emotional disorders in adolescents (UP-A) adapted as a school-based anxiety and depression prevention program: Study protocol of a cluster randomized controlled trial. JMIR Research Protocols, 6(8), e149.

García-Escalera, J., Valiente, R. M., Sandín, B., Ehrenreich-May, J., Prieto, A., \& Chorot, P. (2020). The unified protocol for transdiagnostic treatment of emotional disorders in adolescents 
(UP-A) adapted as a school-based anxiety and depression prevention program: An initial cluster randomized wait-listcontrolled trial Behavior Therapy. Available online at www. sciencedirect.com; doi: https://doi.org/10.1016/j.beth.2019. 08.003

González-Robles, A., García-Palacios, A., Baños, R., Riera, A., Llorca, G., Traver, F., ... \& Botella, C. (2015). Effectiveness of a transdiagnostic internet-based protocol for the treatment of emotional disorders versus treatment as usual in specialized care: study protocol for a randomized controlled trial. Trials, $16,488$.

Grill, S. S., Castañeiras, C., \& Fasciglione, M. P. (2017). Aplicación grupal del Protocolo Unificado para el tratamiento transdiagnóstico de los trastornos emocionales en población argentina. Revista de Psicopatología y Psicología Clínica, 22, 171-181.

Hedges, L. V. (1981). Distribution theory for Glass's estimator of effect size and related estimators. Journal of Educational Statistics, 6, 107-128.

Houck, P. R., Spiegel, D. A., Shear, M. K., \& Rucci, P. (2002). Reliability of the self-report version of the panic disorder severity scale. Depression and Anxiety, 15, 183-185.

Hubbarb, A., Ahern, J., Fleisher, N., Vean Der Laan, M., Lippman, S., Jewell, N.,Cruckner, T., \& Satariano, W. (2010). To GEE or not to GEE: Comparing population average and mixed models for estimating the associations between neighborhood risk factors and health. Epidemiology, 21, 467-474.

Kazdin, A. E., \& Blase, S. L. (2011). Rebooting psychotherapy research and practice to reduce the burden of mental illness. Perspectives on Psychological Science, 6(1), 21-37.

Kennedy, S. M., Bilek, E. L., \& Ehrenreich-May, J. (2019). A randomized controlled pilot trial of the unified protocol for transdiagnostic treatment of emotional disorders in children. Behavior Modification, 43, 330-360.

Kennedy, S. M., \& Ehrenreich-May, J. (2016). Assessment of emotional avoidance in adolescents: Psychometric properties of a new multidimensional measure. Journal of Psychopathology and Behavioral Assessment, 39, 279-290.

Kessler, R. C., Avenevoli, S., Costello, E. J., Georgiades, K., Green, J. G., Gruber, M. J., .. \& Sampson, N. A. (2012). Prevalence, persistence, and sociodemographic correlates of DSM-IV disorders in the National Comorbidity Survey Replication Adolescent Supplement. Archives of General Psychiatry, 69, 372-380.

La Greca, A. M., \& Stone, W.L. (1993). Social Anxiety Scale for Children-Revised: Factor structure and concurrent validity. Journal of Clinical Child Psychology, 22, 17-27.

Mansell, W., Harvey, A., Watkins, E., \& Shafran, R. (2009). Conceptual foundations of the transdiagnostic approach to CBT. Journal of Cognitive Psychotherapy: An International Quarterly, 23, 6-19.

Mineka, S., Watson, D., \& Clark, L. A. (1998). Comorbidity of anxiety and unipolar mood disorders. Annual Review of Psychology, 49, 377-412.

Molina, J., Sandín, B., y Chorot, P. (2014). Sensibilidad a la ansiedad y presión psicológica: Efectos sobre el rendimiento deportivo en adolescentes. Cuadernos de Psicología del Deporte, 14, 45-54.
Moriana, J. A., y Martínez, V. A. (2011). La psicología basada en la evidencia y el diseño y evaluación de tratamientos psicológicos eficaces. Revista de Psicopatología y Psicología Clínica, 16, 81-100.

Nathan, P. E., \& Gorman, J. M. (Eds.). (2015). A guide to treatments that work. Oxford University Press.

Newby, J. M., McKinnon, A., Kuyken, W., Gilbody, S., \& Dalgleish, T. (2015). Systematic review and meta-analysis of transdiagnostic psychological treatments for anxiety and depressive disorders in adulthood. Clinical Psychology Review, 40, 91-110.

Norman, S. B., Hami Cissell, S., Means-Christensen, A. J., \& Stein, M. B. (2006). Development and validation of an overall anxiety severity and impairment scale (OASIS). Depression and Anxiety, 23, 245-249.

Pascual-Vera, B., y Belloch, A. (2018). El carácter transdiagnóstico de las intrusiones mentales: Una revisión y una propuesta basada en datos. Revista de Psicopatología y Psicología Clínica, 23, 135-147.

Pineda, D., Valiente, R. M., Chorot, P., Piqueras, J. A., y Sandín, B. (2018). Invarianza factorial y temporal de una versión española del Cuestionario de Regulación Emocional ERQ. Revista de Psicopatología y Psicología Clínica, 23, 109-120.

Piqueras J. A., Martín-Vivar, M., Sandín, B., San Luis, C., y Pineda, D. (2017). The Revised Child Anxiety and Depression Scale: A systematic review and reliability generalization meta-analysis. Journal of Affective Disorders, 218,153-169.

Piqueras, J. A., Pineda, D., Martín-Vivar, M., \& Sandín, B. (2017). Confirmatory factor analysis and sychometric properties of the Revised Child Anxiety and Depression Scale-30 (RCADS-30) in clinical and non-clinical samples. Revista de Psicopatología y Psicología Clínica, 22, 183-196.

Queen, A. H., Barlow, D. H., \& Ehrenreich-May, J. (2014). The trajectories of adolescent anxiety and depressive symptoms over the course of a transdiagnostic treatment. Journal of Anxiety Disorders, 28, 511-521.

Rapee, R. M., Wignall, A., Sheffield, J., Kowalenko, N., Davis, A., McLoone, J., \& Spence, S. H. (2006). Adolescents' reactions to universal and indicated prevention programs for depression: Perceived stigma and consumer satisfaction. Prevention Science, 7, 167-177.

Rosique, M. T., Chorot, P., Valiente, R. M., García-Escalera, J., y Sandín, B. (2019). Eficacia del Protocolo Unificado (UP) en los trastornos emocionales: Ensayo clínico controlado con relajación y mindfulness como grupos control. En Libro de capítulos: IV Congreso Nacional de Psicología (pp. 125-131). Madrid: Consejo General de Psicología.

Rosique, M. T., Sandín, B., Valiente, R. M., García-Escalera, y Chorot, P. (2019). Tratamiento transdiagnóstico de un caso clínico con elevada comorbilidad de trastornos emocionales: Aplicación del Protocolo Unificado. Revista Argentina de Clínica Psicológica, 28, 79-91.

Sakiris, N., \& Berle, D. (2019). A systematic review and metaanalysis of the unified protocol as a transdiagnostic emotion regulation based intervention. Clinical Psychology Review, $72,101751$.

Sánchez-Arribas, C., Chorot, P., Valiente, R. M., y Sandín, B. (2015). Evaluación de factores cognitivos positivos y negativos relacionadas con el trastorno de pánico: Validación del CATP. Revista de Psicopatología y Psicología Clínica, 20, 85-100. 
Sandín B. (1997). Ansiedad, miedos y fobias en niños y adolescentes. Madrid, España: Dykinson.

Sandín, B. (2003). Escalas PANAS de afecto positivo y negativo para niños y adolescentes (PANASN). Revista de Psicopatología y Psicología Clínica, 8, 173-182.

Sandín, B. (2010). Panic Disorder Severity Scale-Self Report (PDSS-SR) (adaptación española). En B. Sandín (Ed.), Trastorno de pánico. Madrid: UNED.

Sandín, B. (2012). Transdiagnóstico y psicología clínica: Introducción al número monográfico. Revista de Psicopatología y Psicología Clínica, 17, 181-184.

Sandín, B., Chorot, P., Santed, M. A., y Valiente, R. M. (2002). Análisis factorial confirmatorio del Índice de Sensibilidad a la Ansiedad para Niños. Psicothema, 14, 333-339.

Sandín, B., Chorot, P., y Valiente, R. M. (2010). Cuestionario PSWQ-11 para Niños y Adolescentes (PSWQN-11). Universidad Nacional de Educación a Distancia (no publicado).

Sandín, B., Chorot, P., y Valiente, R. M. (2012). Escala de Satisfacción con la Vida para Niños y Adolescentes (SWLSN). Universidad Nacional de Educación a Distancia (no publicado).

Sandín, B., Chorot, P., y Valiente, R. M. (2012). Transdiagnóstico: Nueva frontera en psicología clínica. Revista de Psicopatología y Psicología Clínica, 17, 185-203.

Sandín, B., Chorot, P., y Valiente, R. M. (2016). TCC de los trastornos de ansiedad: Innovaciones en niños y adolescentes. Madrid: Klinik.

Sandín, B., Chorot, P., y Valiente, R. M. (2018). Trastornos de ansiedad en niños y adolescentes. En J. C. Arango-Lasprilla, I. Romero, N. Hewitt-Ramírez, y W. Rodríguez (Eds.), Trastornos psicológicos y neuropsicológicos en la infancia y la adolescencia (pp. 119-161). Bogotá: Manual Moderno.

Sandín, B., Chorot, P., Valiente, R. M., y Chorpita, B. F. (2010). Development of a 30-ítem version of the Revised Child Anxiety and Depression Scale. Revista de Psicopatología y Psicología Clínica, 15, 165-178.

Sandín, B., Chorot, P., Valiente, R. M., y Lostao, L. (2009). Validación española del cuestionario de preocupación PSWQ: Estructura factorial y propiedades psicométricas. Revista de Psicopatología y Psicología Clínica, 14, 107-122.

Sandín, B., Chorot, P., Valiente, R. M., Santed, M. A., y SánchezArribas, C. (1999). Estructura factorial de la Escala de Ansiedad Social para Niños-Revisada (SASC-R). Revista de Psicopatología y Psicología Clínica, 4, 103-113.
Sandín, B., Valiente, R. M., Chorot, P., Santed, M. A., y SánchezArribas, C. (1999). Escala de Ansiedad Social para NiñosRevisada (SASC-R): Fiabilidad, validez y datos normativos. Análisis y Modificación de Conducta, 25, 827-847.

Sandín, B., Valiente, R. M., Sánchez-Arribas, C., y Chorot, P. (2013). Cuestionario de Depresión para Niños y adolescentes $(C D N)$ : Construcción y propiedades psicométricas. Ponencia en III Symposium Nacional de Psicología Clínica y de la Salud en Niños y Adolescentes. Elche (Valencia), 13-15 de noviembre.

Sauer-Zavala, S., Gutner, C. A., Farchione, T. J., Boettcher, H. T., Bullis, J. R., \& Barlow, D. H. (2017). Current definitions of «transdiagnostic» in treatment development: A search for consensus. Behavior Therapy, 48, 128-138.

Shear, M. K., Brown, T. A., Barlow, D. H., Money, R., Sholomskas, D. E., Woods, S. W., ... \& Papp, L. A. (2006). Multicenter collaborative panic disorder severity scale. American Journal of Psychiatry, 154, 1571-1575.

Sheehan, D.V., Lecrubier, Y., Shytle, D., Milo, K., Hergueta, T., Colón-Soto, M., Díaz, V., \& Soto, O. (2000). Mini International Neuropsychiatric Interview for children and adolescents (MINIKID ). Version 1.1. Tampa: Medical Outcome Systems, Inc.

Silverman, W. K., Fleisig, W., Rabian, B., \& Peterson, R. A. (1991). Childhood anxiety sensitivity index. Journal of Clinical Child and Adolescent Psychology, 20, 162-8.

Titov, N., Dear, B. F., Johnston, L., \& Terides, M. (2012). Transdiagnostic internet treatment for anxiety and depression. Revista de Psicopatología y Psicología Clínica, 17, 237-260.

Valiente, R. M., Sandín, B., y Chorot, P. (2002). Miedos comunes en niños y adolescentes: Relación con la sensibilidad a la ansiedad, el rasgo de ansiedad, la afectividad negativa y la depresión. Revista de Psicopatología y Psicología Clínica, 7 , 61-70.

Valiente, R. M., Sandín, B., y Chorot, P. (2003). Miedos en la infancia y la adolescencia. Madrid, España: UNED.

Vigerland, S., Lenhard, F., Bonnert, M., Lalouni, M., Hedman, E., Ahlen, J., .. \& L Lótsson, B. (2016). Internet-delivered cognitive behavior therapy for children and adolescents: A systematic review and meta-analysis. Clinical Psychology Review, 50, 1-10.

Wittchen, H. U., Jacobi, F., Rehm, J., Gustavsson, A., Svensson, M., Jönsson, B., .. \& \& Fratiglioni, L. (2011). The size and burden of mental disorders and other disorders of the brain in Europe 2010. European Neuropsychopharmacology, 21, 655-679. 